\section{Infection Control Measures in an Israeli District Hospital Receiving Syrian Civil War Casualties Colonized with Multi-Drug Resistant Bacteria}

\section{Seema Biswas ${ }^{1 *}$, Amram Hadary ${ }^{1}$, Shorooq Aladdin ${ }^{1}$, Hagai Rechnitzer $^{2}$, Shokrey Kassis ${ }^{3}$, Daniel Glikman ${ }^{4,5}$, Evgeny Solomonov $^{1}$, Igor Waksman ${ }^{6}$ and Dan Miron ${ }^{7}$}

${ }^{1}$ Department of Surgery, Ziv Medical Center, Safed, Galilee, Israel ${ }^{2}$ Clinical Microbiology Laboratory, Ziv Medical Center, Safed, Galilee, Israel ${ }^{3}$ Department of Plastic Surgery, Ziv Medical Center, Safed, Galilee, Israel ${ }^{4}$ Pediatric Infectious Diseases Service, Galilee Medical Center, Nahariya, Israel

${ }^{5}$ The Faculty of Medicine, Bar-llan University, Safed, Israel

${ }^{6}$ Department of Surgery, Western Galilee Hospital, Nahariya, Galilee, Israel

${ }^{7}$ Infectious Diseases Consultation Service, Ziv Medical Center, Safed, Galilee, Israel

\section{Background}

As the Syrian civil war continues, at least 4 million people have fled the country and 220,000 people are estimated to have been killed over the last four years [1]. There has been widespread destruction of infrastructure, including devastation of healthcare services, resulting in the resurgence of communicable diseases such as polio and leishmaniasis [2], and a lack of basic surgical care. Over the last two and a half years, Ziv Medical Center, a 320 bed hospital, in Northern Israel, close to the border with Lebanon and Syria has received over 450 Syrian casualties of the civil war. An alarming finding has been the culture of various Multi-Drug Resistant (MDR) bacteria from Syrian patients admitted to several hospitals in Israel [3]. These bacteria include Methicillin-resistant Staphylococcus aureus (MRSA), MDR Acinetobacter sp., Extended-spectrum beta-lactamase (ESBL)-producing Enterobacteriaceae and Carbapenem-resistant Enterobacteriaceae (CRE). Of the first 306 patients admitted to Ziv Medical Center between February 2013 and May 2014, 40.8\% patients

${ }^{*}$ Corresponding author: Seema Biswas, Department of Surgery, Ziv Medical Center, Safed, Galilee 13100, Israel, Tel: +972 504327252; E-mail: seemabiswas@msn.com

Citation: Biswas S, Hadary A, Aladdin S, Rechnitzer H, Kassis S, et al. (2015) Infection Control Measures in an Israeli District Hospital Receiving Syrian Civi War Casualties Colonized with Multi-Drug Resistant Bacteria. J Infect Non Infect Dis 1: 004.

Received: May 05, 2015; Accepted: Jun 09, 2015; Published: Jun 23, 2015 were found to have MDR (including ESBL) from bacterial cultures (including surveillance cultures for MDR) taken within 3 days of admission, and $70 \%$ of patients had MDR (including ESBL) from all cultures during their period of admission to Ziv. The consequences to individual patients suffering life-threatening infections, to admitting health facilities in all countries neighbouring Syria and to countries receiving refugees more widely, including Europe, is an alarming clinical and public health threat.

Preliminary results from the Galilee (Nahariya) and Baruch Padeh (Poriya) Medical Centers in Israel [3] indicate an approximately $40 \%$ carriage rate amongst Syrian adults of various multi-drug resistant organisms including MRSA, MDR A. baumannii, carbapenem-resistant Enterobacteriaceae; and, an approximately $70 \%$ carriage rate of Extended-spectrum beta-lactamases (ESBL) among Syrian children. Quoting Peretz et al. [3], 19 out of 29 children (66\%) treated at the hospitals were found to have MDR. "ESBL comprised 20 of 21 MDR isolates (19/20 were Escherichia coli); 1 isolate was MRSA. Among the adult patients, 28 of 60 (47\%) carried MDR bacteria. In adults, however, the types of MDR isolates differed: CRE carriage was noted (5 patients, 2 with New Delhi metallo- $\beta$-lactamase), as well as MRSA".

Much needs to be done in order to discover the origins and mechanisms of antibiotic resistance, but initial findings indicate high rates of ESBL carriage amongst children (most of whom have not been previously hospitalised in Syria) and who are likely, therefore, to have community-acquired carriage of resistant organisms [3-5].

Research within Syria has shown the alarming level of antibiotic resistance there before the present civil conflict began [6,7]. Causes of resistance include the liberal use of antibiotics from poor prescribing where clinical indications are weak, to over-the-counter distribution of drugs without medical prescriptions [7-9]. In addition, the industrial use of antibiotics, in farming, for example, facilitates the development and propagation of resistance $[10,11]$. Syrian scientific literature indicates that this has been a cause of concern for some time [12]. It is possible, that this has been further compounded by the collapse of large sections of the Syrian healthcare system.

The purpose of this paper is to outline infection control measures necessary within the hospital taken in order to reduce the incidence and duration of infections amongst the patients from Syria, to prevent cross infection and to form a cohesive plan of action across multiple departments under challenging circumstances.

\section{Problem}

Routine infection control measures in Ziv Medical Center include the isolation of all patients transferred to the hospital from other health facilities, with microbiological screening cultures taken at admission. Policy then dictates that patients are kept in isolation. This has been a major challenge with Syrian patients, not simply because of the volume of patients received in the hospital, but also because all hospital departments, including the pediatric and adult intensive care units, have been involved in the care of the patients and almost all the patients have required surgery. 
Some patients arrived after receiving basic paramedical care or medical treatment in a field hospital. Others arrived up to five days after initial injury, with infected wounds that had been sutured primarily after trauma in Syrian health facilities, with improvised, soiled dressings and dirty clothes and blankets. The number of septic complications amongst the first 100 patients admitted to Ziv Medical Center is shown in table 1 . Table 2 shows the causative organisms of infections amongst the first 100 patients treated in Ziv Medical Centre. Twenty two patients were found to have MDR bacteria, including ESBL, MRSA, CRE and Acinetobacter.

\begin{tabular}{|c|c|}
\hline Complication & Number of patients \\
\hline Abdominal collection & 1 \\
\hline Wound infection & 7 \\
\hline Septicemia & 6 \\
\hline Pneumonia & 9 (4 with Klebsiella pneumoniae $)$ \\
\hline Lung abscess & 1 \\
\hline
\end{tabular}

Table 1: Infective complications amongst first 100 patients treated in Ziv Medical Center (not all patients had resistant organisms).

\begin{tabular}{|c|c|}
\hline Organism & Number of patients \\
\hline Coagulase negative Staphylococcus & 2 \\
\hline Escherichia coli & 5 \\
\hline Acinetobacter spp. & 3 \\
\hline Enterobacter spp. & 4 \\
\hline Pseudomonas spp. & 2 \\
\hline Klebsiella spp. & 3 \\
\hline MRSA & 1 \\
\hline
\end{tabular}

Table 2: Pathogenic organisms as causes of bacteraemia in Ziv Hospital amongst first 100 patients (not all organisms display resistance).

\section{Intervention}

As a consequence of the discovery of multi-drug resistance and the high incidence of septic wounds and complications amongst the first cohort of patients occupying the ICU, infection control policy across the hospital has involved isolation of patients from their admission to discharge, special infection control measures in the operating theatres, a unique antibiotic policy and recruitment of staff to nurse patients in isolation.

\section{Isolation}

Patients are admitted via the trauma room (almost all patients have multiple injuries) and remain there until ready to proceed either to the operating theatre or to the radiology department, intensive care units or wards. In practice, most patients proceed directly to the operating theatre but patients undergoing radiological investigation return to the trauma room unless ready for surgery or the ward.

This is at odds with the policy to keep the four-bed trauma room free in readiness for the next emergency but is a necessary measure to avoid contamination of the rest of the emergency department.

On the wards, patients remain in contact isolation in single rooms, double rooms or bays. Only essential medical and nursing staffs are permitted to enter their rooms and they must gown and glove before they do so. It is impossible to understand just how this must affect patients already traumatised by war, far from home and their loved ones, in a foreign country (considered a hostile country) and unable to converse in Hebrew with many of their care-givers. Where possible, relatives (including non-injured companions) are placed together but this, in itself, is an infection control compromise and cross-infection is a very real risk. Books, games, computers, radios and televisions have been placed in all the rooms and the bays and an Arabic speaking social worker visits daily.

\section{Protocol in the operating theatres}

There are two large recovery areas in the operating theatre complex. Only one of these is used for patients in isolation. Of necessity, both Orthopaedic and General Surgery Operating Theatres are used for patients but, isolation patients have their own operating table, and in Orthopaedics, their own disposable drills. Theatres are cleaned after every case (with double the usual dose of chlorine tablets dissolved in water ( $2 \mathrm{~g}$ tablets dissolved in 1 litre of water as opposed to $1 \mathrm{~g}$ (1000 ppm - the standard dilution), and disposable equipment is incinerated in the standard way. All surgical instruments are put through the standard sterilisation procedures comprising two enzymatic wash cycles (liquizime) and gamma ray sterilisation.

\section{Nursing}

All hospital personnel involved in the care of patients are subject to hospital infection control measures. These measures are reinforced in training sessions and by the infection control team of the hospital. Medical staff and cleaning staff, porters, and visitors wear gowns and gloves and theatre masks, and dispose of all potentially contaminated material in bins provided at the door. Alcohol gel and hand-washing facilities are available by the door.

For patients in need of individualised care, nurses must be contracted to look after patients for all shifts. This is expensive but a necessary measure in both clinical care and infection control.

\section{Surveillance and other cultures obtained on admission}

Surveillance cultures for MRSA are obtained from each patient, from the nasal, axillary and perianal area, for ESBL, VRE (Vancomycin Resistant Enterococci), CRE, and Acinetobacter sp. from the stool, wounds and tracheal aspirates. Other cultures are obtained based on the clinical situation and findings of surgery.

\section{Antibiotic policy}

Culture results with positive results for MDR are routinely flagged on the hospital computer system. The standard antimicrobial policy in the hospital for all Syrian patients initially admitted includes treatment with intravenous Amikacin and Vancomycin, (a regimen based on our practice for treating ESBL, MRSA and MDR bacteria), metronidazole (when anaerobic bacterial coverage is needed) for 48-72 hours, and passive and active tetanus immunization (as patients are unable to confirm previous tetanus immunization). Thereafter, antimicrobial therapy is guided by the results of bacterial cultures obtained based on the clinical situation.

\section{Summary}

Clinical and epidemiological data is essential in order to understand the scale of antibiotic resistance within Syria and the Middle East. It stands to reason that the disruption of health services in Syria is likely to have further compounded the spread of multi-drug resistance and efforts to monitor and contain this are important.

Strict infection control policies are notoriously difficult to enforce and their effectiveness warrants regular evaluation. Physical isolation compounds emotional isolation and the effects of infection control measures that affect social contact and social well-being need to be studied. 


\section{Conclusion}

As further data emerges about the pathogens involved in infections amongst these patients, the origins of regional multi-drug resistance, carriage rates of resistant organisms and practices that increase resistance in these communities, clinical and public health measures will need to be addressed. Measures are necessary to control the spread of resistance amongst populations that, of necessity, have fled their homes and are in constant movement across the world.

\section{References}

1. Rodgers L, Gritten D, Offer J, Asare P (2015) Syria: the story of the conflict. BBC News, Middle East.

2. Sharara SL, Kanj SS (2014) War and infectious diseases: challenges of the Syrian civil war. PLoS Pathog 10: 1004438.

3. Peretz A, Labay K, Zonis Z, Glikman D (2014) Disengagement does not apply to bacteria: a high carriage rate of antibiotic-resistant pathogens among Syrian civilians treated in Israeli hospitals. Clin Infect Dis 59: 753-754.

4. Biswas S, Waksman I, Baron S, Fuchs D, Rechnitzer H, et al. (2015) Analysis of the First 100 Patients From the Syrian Civil War Treated in an Israeli District Hospital. Ann Surg. Apr 17.

5. Gilbey P, Spivey SE (2015) Healing the enemy: the personal narrative of an Israeli surgeon. Ann Intern Med 162: 389-390.
6. WHO (2014) Antimicrobial resistance: Global report on surveillance. WHO, USA.

7. Al-Assil B, Mahfoud M, Hamzeh AR (2013) Resistance trends and risk factors of extended spectrum $\beta$-lactamases in Escherichia coli infections in Aleppo, Syria. Am J Infect Control 41: 597-600.

8. Al-Faham Z, Habboub G, Takriti F (2011) The sale of antibiotics without prescription in pharmacies in Damascus, Syria. J Infect Dev Ctries 5: 396-399.

9. Otoom SA, Sequeira RP (2006) Health care providers' perceptions of the problems and causes of irrational use of drugs in two Middle East countries. Int J Clin Pract 60: 565-570.

10. Barah F, Gonçalves V (2010) Antibiotic use and knowledge in the community in Kalamoon, Syrian Arab Republic: a cross-sectional study. East Mediterr Health J 16: 516-521.

11. Hong P-Y, Al-Jassim N, Ansari MI, Mackie RI (2013) Environmental and Public Health Implications of Water Reuse: Antibiotics, Antibiotic Resistant Bacteria, and Antibiotic Resistance GeneA. Antibiotics 2: 367-399.

12. Feldgarden $M$ (2010) Resistance in Asian and Middle Eastern Commensals. In: de J Sosa A, Byarugaba DK, Amabile C, Hsueh P-R, Kariuki S, et al. (eds.). Antimicrobial resistance in developing countries. Springer, London, UK. Pg no: 271. 